\title{
VALORACIÓN DEL DESARROLLO CONCEPTUAL Y DE LAS HABILIDADES PROCEDIMENTALES Y DE RAZONAMIENTO EN ESTUDIANTES DEL CURSO DE TECNOLOGÍA DE CEREALES EN UNAD PASTO, CON LA UTILIZACIÓN, COMO MEDIADOR DIDÁCTICO, DE UN SIMULADOR EN PROCESOS DE PANIFICACIÓN
}

\author{
Fedra Lorena Ortiz, ${ }^{74}$ Clemencia Álava, ${ }^{75}$ \\ Magali Argoty, ${ }^{76}$ Pablo Fernández ${ }^{77}$
}

\begin{abstract}
RESUMEN
Las estrategias de aprendizaje se han aplicado en los últimos años en diferentes contextos educativos; varios estudios demuestran que cuando se aplican, los estudiantes mejoran su aprendizaje. Por otra parte, con el desarrollo de nuevas tecnologías se ha presentado a los Objetos virtuales de aprendizaje como una alternativa para el desarrollo de habilidades cognitivas. Sin embargo, Carreras (2003) afirma que este tipo de objetos no alcanza lo deseado si no están acompañados de estrategias de enseñanza-aprendizaje que orienten el proceso. Por tanto, la pregunta que guió esta investigación fue: ¿Los estudiantes aprenden mejor los conceptos y mejoran sus habilidades procedimentales y de razonamiento, cuando utilizan un simulador, a través de una estrategia de aprendizaje o cuando lo utilizan sin estrategia?
\end{abstract}

Esta investigación se desarrolló en dos fases. En la primera se diseñó un simulador para el proceso de panificación y se estructuró una propuesta metodológica basada en la estrategia ABP (Aprendizaje Basado en Problemas). En la segunda se planteó un diseño cuasi experimental, con prueba postest y pretest con un grupo experimento y un grupo control, diseño que se aplicó a estudiantes del curso de tecnología de cereales y oleaginosas que se ofrece en el programa de ingeniería de alimentos; estos cuestionarios se dividieron por bloques de competencia de aprendizaje así: conceptual, procedimental y de razonamiento. Para el tratamiento de los resultados se aplicó una prueba o contraste de signos y el test de Kluster Wallis.

Palabras clave: estrategia de aprendizaje, simulador, TIC, desarrollo conceptual, habilidades de razonamiento, habilidades procedimentales.

\footnotetext{
ABSTRACT

The learning strategies have been applied in the past years in different educational contexts; several studies show that when they are put in use, the students improve their learning. On the other hand, with the development of new technologies, the virtual learning objects have

74 Docente Auxiliar Escuela de Ciencias Básicas Tecnología e Ingeniería. UNAD Pasto.

75 Decana espejo Escuela de Ciencias Básicas Tecnología e Ingeniería. Zona Centro Sur UNAD.

76 Estudiante del programa de Ingeniería de sistemas. UNAD Pasto.

77 Docente Asistente Programa de Biología. UDENAR.
} 
been presented as an alternative for the development of cognitive abilities. Nonetheless, Carreras (2003) affirms that these kinds of objects do not reach the expectations if they are not accompanied by teaching-learning strategies that guide the process. Because of this, the question that ruled the investigation was: Do the students learn the concepts better and improve their procedure and reasoning abilities when they use a simulator guided by a learning strategy or when this is use without it?

The research was developed in two phases. In the first phase a simulator for the process of making bread was developed and a methodological proposal was structured based in the ABP strategy (Aprendizaje Basado en Problemas, Learning based in problem solving). In the second phase, a quasi experimental design was proposed, with a posttest and pretest sample with an experiment group and a control group, The design was applied to students of the Grain Technology and Oleaginous class offered by the Food Engineering program; these samples were divides between the sectors of learning competencies in the following way: conceptual, procedure, and reasoning. For the treatments of the results, a test or sign contrast, and the Kluster Wallis test were used.

Key words: Learning strategy, simulator, Tic, conceptual development, reasoning abilities, procedure abilities.

Recibido: 16 de marzo de 2009

Aceptado: 17 de abril de 2009

\section{INTRODUCCIÓN}

La Universidad Nacional Abierta y a Distancia (UNAD) es la única universidad pública de Colombia que ofrece todos sus programas en la modalidad a distancia; esta modalidad educativa se caracteriza por la aplicación de la tecnología del aprendizaje sin las limitaciones del lugar, de tiempo, ocupación o edad de los estudiantes (Silvestre, 1999; Zapata, 2002; Galvis, 2003), surge como una necesidad del desarrollo y constituye una alternativa condicionada por las características de los alumnos a quienes va dirigida. La educación a distancia se entiende, en términos dobles, como una modalidad y una estrategia educacional en la cual existen dos rasgos distintivos esenciales (Zapata 2002):

- El primero es la mediatización del acto pedagógico, es decir, la separación del momento en que se produce la enseñanza, del momento en que se inicia el aprendizaje.

- El segundo es la sustentación de la educación a distancia en un soporte tecnológico, el que ha variado y evolucionado con el tiempo: libros, radio, televisión, video, recursos multimedia. Sin este soporte no es posible desarrollar la educación a distancia, por consiguiente el impacto que ha tenido el desarrollo de las telecomunicaciones en este tipo de educación más que en la educación presencial, no tiene precedentes. 
Sin embargo, García (2006) advierte que la aplicación de tecnologías educativas puede concebir en los educadores una interpretación artefactual economicista del uso de las tecnologías en la educación, conformándose sólo con la instalación de estos artefactos en el aula o transformando la web en un depósito de documentos. Por el contrario, señala este autor, se trata de pasar a desarrollar competencias tecnológicas-culturales específicas entre los integrantes de la comunidad educativa.

Fainholc (2004) agrega además que con el aparecimiento de las TIC en el proceso de enseñanza/aprendizaje es posible fundamentar una tecnología educativa crítica que sobre la base de un enfoque holista, interactivo y constructivo, se incorpore en el proceso educativo en forma reflexiva, contextualizada y estratégica que garantice el respeto a la diversidad, multiculturalidad y la convivencia solidaria.

Esteban, M. (2002) complementa que el mismo dinamismo social promoverá nuevas formas de aprender, nuevas habilidades; la escuela y la universidad habrán de abrirse más — mucho más- a las condiciones y prescripciones de un mundo permeable e instantáneo en el acceso a la información pero más complejo y especializado en la selección de la misma. En este sentido, González (2005) considera que estos son los tiempos de la Educación a Distancia como una forma de enfrentar esta nueva sociedad del aprendizaje puesto que tiene diversas significaciones como aprendizaje abierto, en casa, autoestudio guiado, estudiar sin dejar de producir, conversación didáctica guiada y es una forma de autodidaxia asistida que permite al estudiante adulto acceder a fuentes mediatizadas de saberes, sin la intervención clásica de un profesor, pero con el apoyo de una red de recursos que encuadran su aprendizaje; (Eisner,1994; Esteban M., 2002). En efecto, la educación a distancia amplía significativamente el acceso a la educación sin las tradicionales limitaciones de tiempo y espacio, y con oportunidades de mayor flexibilidad en los procesos cognitivos.

A pesar de ello, uno de los inconvenientes más difíciles de resolver en este tipo de modalidad son los laboratorios o experimentaciones requeridas para los cursos académicos que se ofrecen. Entre las alternativas para dar solución a este tipo de situación está el realizar convenios con instituciones cercanas al lugar de vivienda de los estudiantes, para que puedan hacer en ellas sus prácticas o, el diseñar software educativo que permitan simular los experimentos requeridos en los cursos, sin embargo existe una fuerte controversia frente a la efectividad del aprendizaje con simuladores. Zapata (2002).

Los simuladores, se definen como objetos de aprendizaje que mediante un programa de software intentan revelar parte de una réplica de los fenómenos de la realidad y el propósito es que el usuario construya conocimiento a partir del trabajo exploratorio, la inferencia y el aprendizaje por descubrimiento. Los simuladores se desarrollan en un entorno interactivo, que permite al usuario modificar parámetros y ver cómo reacciona el sistema ante el cambio producido. Mortimer (2007).

Entre las ventajas de utilizar simuladores (Hilera, 1999; Marqués, 2000; García, 2006) se encuentra que el estudiante puede hacer sus primeras prácticas y cometer errores en un objeto 
inanimado donde, en el peor de los casos, al cometer un error grave lo único que hay que hacer es reiniciar la práctica para mejorar la destreza y evitar los errores. Señalan que el manejo apropiado de una situación de emergencia es muy difícil de enseñar en ambientes de educación presencial. La única manera de practicar y que el estudiante aprenda de los errores es en un ambiente simulado sin necesidad de poner en peligro al aprendiz o a sus compañeros. Otra ventaja de los ambientes simulados es permitir que los alumnos observen entidades que, por su rara aparición, difícilmente verán durante sus estudios o que puedan manejar maquinaria a la que muy seguramente no pueden tener acceso por los altos costos. Por otra parte, Pontes (2005) afirma que estos objetos pueden ser de gran interés en la educación científica y técnica por las posibilidades que ofrece el ordenador desde el punto de vista de la comunicación, la interactividad, el tratamiento de imágenes, la simulación de fenómenos y experimentos, la construcción de modelos, la resolución de problemas, el acceso a la información y el manejo de todo tipo de datos.

Sin embargo, estos objetos de aprendizaje no están exentos de críticas, ya que existe una aparente desconexión entre el carácter generalmente instruccional de estos materiales y las tendencias actuales en educación que enfatizan la importancia del aprendizaje colaborativo y de las comunidades de aprendizaje. Algunos autores (López, M., 2000; Coll, 1990; Gargallo, 2000) coinciden en advertir que el uso de estos objetos sin una estrategia pedagógica puede llevar a un aprendizaje mecanicista en que los estudiantes repiten los procesos sin activar sus conocimientos previos que los impulsen a un verdadero aprendizaje significativo $\mathrm{y}$, por el contrario, estimulan el conductismo, en su modelo más elemental en el esquema E-R (Estímulo-Respuesta). Rojas (2005).

Por consiguiente, muchas han sido las estrategias propuestas para salvar esta situación. Una de las más utilizadas en la educación a distancia es el aprendizaje colaborativo donde el tutor juega un rol muy importante, de guía adecuada para que los aprendices interactúen entre sí con los materiales liberados en la web, y busquen en la red la experiencia de expertos investigadores que les ayuden a completar sus conocimientos previos para generar nuevos conocimientos, Varela (1997). Una de las aplicaciones de este tipo de estrategias es el Aprendizaje Basado en Problemas (ABP). Este es un modelo con enfoque constructivista, que evolucionó a partir de los trabajos de psicólogos y educadores tales como Lev Vigotsky, Jerome Bruner, Jean Piaget y John Dewey. En este enfoque son los estudiantes quienes planean, implementan y evalúan situaciones que pueden llevar a resolver un determinado problema que ha sido planteado previamente por el docente.

En atención a los anteriores planteamientos, en este trabajo se evaluaron el desarrollo conceptual y las habilidades procedimentales, y de razonamiento, en estudiantes del curso de tecnología de cereales de la UNAD - CEAD Pasto, utilizando como mediador didáctico un simulador para procesos de panificación. 


\section{METODOLOGÍA}

El desarrollo de la investigación se planteó en dos etapas:

1. Diseño de un simulador para entrenamiento en procesos de panificación. Se construyó a partir del desarrollo de una tesis para optar el título profesional como Ingeniero de Sistemas de la UNAD, con la asesoría de un Ingeniero de Alimentos. Para su desarrollo se partió de una triangulación de la información entre los temas del curso académico de Tecnología de cereales, los objetivos y las competencias que se deseaban alcanzar en el curso y el tipo de objeto de aprendizaje que se deseaba construir, basado en la teoría de Ingeniería de software.

Para el desarrollo de este software se utilizaron la modelación matemática y la simulación como teoría principal; se realizaron diferentes observaciones y cálculos matemáticos, que permitieron predecir el comportamiento del proceso de panificación en la selección de fórmulas utilizadas en la industria: pan de dulce, sal, francés y torta; así como los ingredientes y las etapas del proceso: selección de materias primas de acuerdo a formulación, amasado, reposo, división, fermentación y horneo. El simulador se diseñó para que el usuario pueda acceder a los datos de balance de materia y energía que se obtendrían del proceso planteado; también puede visualizar el comportamiento de las variables tiempo y temperatura las cuales están programadas por rangos. Al finalizar la simulación del proceso, el usuario tiene la posibilidad de observar las características organolépticas del producto y, de esta manera, analizar el procedimiento en todas sus fases, detectar errores y corregirlos. Para el desarrollo del software se utilizó el lenguaje de programación Visual Basic 6.0 y Base de datos en Access 2000.

2. Evaluación de la estrategia ABP, empleando el simulador de procesos panificación como medio didáctico. Para este estudio se planteó un diseño cuasi experimental, con prueba postest y pretest, con un grupo experimento y un grupo control. Como los grupos ya estaban preestablecidos el número de estudiantes en cada grupo varió. El primer grupo estaba conformado por quince estudiantes, mientras el segundo grupo estaba conformado por doce personas. Con el fin de homogenizar las muestras y teniendo en cuenta que en la UNAD, no existen cursos de estudiantes por semestres, el único parámetro que se tuvo en cuenta para el estudio, fue que los estudiantes de los dos grupos hayan visto el curso de tecnología de cereales y oleaginosas y se hayan matriculado en el programa de ingeniería de alimentos de la UNAD- CEAD Pasto, como estudiantes regulares.

Para el primer grupo se realizaron diez sesiones. En la primera sesión se les aplicó el pretest que sirvió de diagnóstico para saber las condiciones en las cuales se encontraban frente a las competencias evaluadas. En la segunda sesión se les dio a conocer el simulador y se les explicó su manejo. En las siguientes debían resolver el problema propuesto en la estrategia de aprendizaje utilizando el simulador; en este sentido, el software les proporcionó varias ayudas didácticas que les permitían profundizar en los conceptos, a la vez que aplicarlos desarrollando los procedimientos propios para panificación $\mathrm{y}$, proponer alternativas de solución al problema planteado. Una vez resuelta y socializada la estrategia utilizando el simulador, los estudiantes respondieron el postest. 
El mismo procedimiento se realizó para el segundo grupo pero, a diferencia del grupo anterior, los estudiantes manejaron el simulador en el que pudieron conocer su funcionamiento y acceder a los diferentes recursos del software como vídeos, infografías, entre otros. Sin embargo, no se les planteó ninguna situación de aprendizaje en la que tuvieran que aplicar los conceptos.

Tanto el cuestionario pretest como el postest se dividieron por bloques de competencia de aprendizaje así: Conceptual, Procedimental y de Razonamiento. Cada bloque estaba constituido por cinco preguntas. Con el fin de que la evaluación fuera lo más objetiva posible, se evitaron las preguntas abiertas y sólo se formularon preguntas cerradas con una o dos alternativas de respuesta. Para evitar que los estudiantes se aprendieran las preguntas de memoria, e incidiera en el resultado del postest, se utilizaron preguntas diferentes en los dos cuestionarios, pero del mismo tema. Con el fin de procesar los datos se dio una calificación de un (1) punto por cada respuesta correcta y cero (0) por respuesta incorrecta, Debido a esto el puntaje final, por cada bloque de preguntas, varió entre cero y cinco.

Para el tratamiento de los resultados se aplicó una prueba no paramétrica de contraste de signos de acuerdo con las características de las muestras de estudio, para la cual se elaboró una prueba de hipótesis unilateral. Ella es:

Ho: Se estableció si la mediana del postest es menor o igual que la mediana del pretest:

Ho: $\eta$ pos test $\leq \eta$ pre test,

Y, cómo hipótesis alterna, se planteó que la mediana del postest es mayor que la mediana del pretest.

Ha; $n$ pos test $>n$ pre test

Una vez definida la prueba estadística, se escribieron las hipótesis de investigación en función de los bloques de competencias evaluadas:

Las hipótesis estadísticas ascendieron a un total de seis (tres por grupo) y, se formularon de acuerdo con los requerimientos de la investigación, según un contraste unilateral derecho con nivel de confianza del $95 \%$. 
Gráfico 1. Hipótesis estadísticas

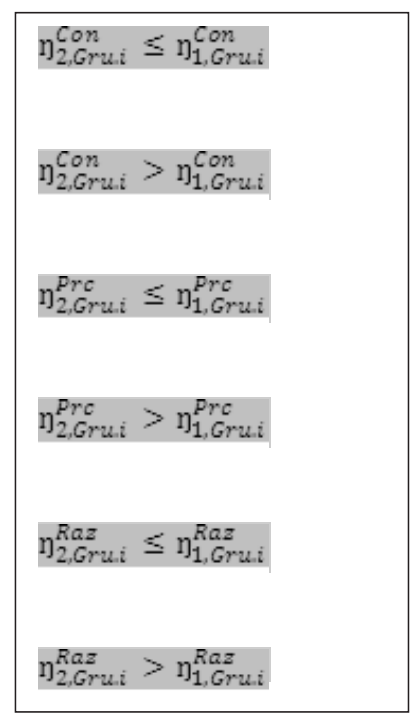

El símbolo $\eta$ significa mediana, y los superíndices CONC, PRC y RAz hacen referencia a los bloques de actividades de conceptos, procedimientos y razonamiento. El subíndice i que puede tomar el valor 1 o 2 concretaría las hipótesis de investigación para uno u otro grupo investigado. Por último los subíndices 1 y 2 se referirían a los valores antes (1) o después (2) de la experiencia. En definitiva, se analizó la efectividad de la estrategia ABP como eje del aprendizaje mediado por las tecnologías de la información y la comunicación. Gómez (2005).

Con el fin de identificar si había diferencias estadísticas significativas en los valores de las medianas de las competencias de aprendizaje evaluadas en el postest del primer grupo, se aplicó el test de Kluster Wallis.

\section{RESULTADOS Y DISCUSIÓN}

El simulador cuenta con varios elementos, como la simulación del proceso, con recursos didácticos que facilitan la comprensión de los procedimientos y, con evaluaciones de desempeño; también, con videos y literatura relacionada con el tema, de manera que se encaminó a facilitar el aprendizaje de las etapas que tienen que ver en el proceso de panificación en la producción industrial automatizada, teniendo en cuenta los siguientes parámetros: la formulación del pan (según el tipo: pan de dulce, pan de sal, y torta comercial), ingredientes, donde el usuario interactúa ingresando variables que le pide el sistema. Luego presenta las etapas de panificación como amasado, reposo, división por unidad de presentación (según el peso), fermentación, horneo donde le da al estudiante a escoger e ingresar variables para el análisis. El simulador está diseñado para que con la interacción, el usuario analice las variables a partir del modelo matemático y proyecte un análisis pormenorizado de las características del producto final. 
Permite interactuar al usuario en la realización del proceso de panificación con los diferentes tipos de pan que se especifican en el simulador como pan de sal, dulce, francés y torta. Al terminar la simulación, por medio de un análisis sistemático, el software indica al usuario los errores o aciertos en el proceso. Para cada tipo de pan, se estructuró un modelo matemático que permite analizar el proceso de acuerdo a criterios específicos, y compara los datos del modelo con la información que el usuario digite. Igualmente, el software genera una imagen con las características del producto final; de la misma forma se presentan los resultados y el análisis de la simulación lo cual permite al usuario detectar fácilmente los errores que se han cometido en el proceso y corregirlos, entrenando al usuario para aplicar el proceso en otro ambiente de aprendizaje.

Durante la exposición al simulador, los dos grupos de estudiantes demostraron facilidad en el manejo y aplicación del software, sin embargo, en el Grupo 1, se observó una mayor interactividad y motivación para trabajar en grupo, coincidiendo con el planteamiento de López (2007) en que la contextualización de estos materiales es responsabilidad de los docentes, en cualquier ambiente donde se realice el proceso de aprendizaje. Es el profesor el responsable de dar sentido pedagógico a estos materiales incorporándolos a sus actividades y utilizando las estrategias didácticas que considere más oportunas. En este sentido, la alta preparación pedagógica de los profesores se convierte en un factor importante que permite hacer del software una herramienta adecuada para alcanzar los objetivos educativos en diferentes niveles y disciplinas; esta podría ser la clave para impulsar la utilización de las TIC en el aula, especialmente, en el ámbito de los procesos científicos.

Una vez aplicada la prueba de signos se contestó la siguiente pregunta estadística:

¿Existen diferencias significativas entre los resultados obtenidos por los estudiantes antes y después del tratamiento, tanto en el Grupo 1, como en el Grupo 2?

El análisis para esta pregunta mostró que en el Grupo 1 hubo mejores resultados en la prueba de postest para los tres tipos de aprendizaje: Conceptos, Procedimientos y Razonamientos. Mientras que el análisis con contraste unilateral izquierdo puso de manifiesto que en el grupo 2 el bloque de conceptos no tuvo diferencia y, por el contrario, los resultados obtenidos en los bloques de preguntas de razonamiento y de procedimiento mostraron una desmejora con relación a la prueba de pretest (ver Tabla 1).

Tabla 1. Resultados de las pruebas objetivas en los grupos 1 y 2

\begin{tabular}{|c|c|c|c|c|c|}
\hline t & VR:LB:5 & Мอนи & & $p$ & MPOTESNIA \\
\hline \multirow[t]{2}{*}{ Besulas B } & & माहता & Postr্口 & (Machnco) & to:flunest) \\
\hline & Concelts & 2 & 4 & 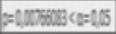 & Sercias \\
\hline \multirow[t]{3}{*}{ GuON } & poesinietss & 1 & 3 & $800105513<0: 105$ & Seretar \\
\hline & Bavanietto & 2 & 4 & $=0.033579<0=0.15$ & Serecias \\
\hline & Concegtss & 3 & 3 & $z=100=010$ & Sasune \\
\hline \multirow[t]{2}{*}{ GuOPB } & Powinietss & 4 & 3 & $z=0.15) 0=015$ & Sarane \\
\hline & Bunanets & 3,5 & 2 & $y=016029)=015$ & Sassene \\
\hline
\end{tabular}

Fuente: Esta investigación 
El mejoramiento en la apropiación conceptual de los temas tratados pudo deberse a que los estudiantes debían recurrir a los recursos didácticos ofrecidos por el software, como videos infografías y literatura lo cual les permitió identificar el concepto, aplicarlo en el simulador para optimizar el manejo del mismo y, obtener resultados satisfactorios; esto demuestra la teoría del aprendizaje significativo, ya que el estudiante dinamizó los conocimientos previos con los recursos del simulador, y esto le permitió resolver el problema, utilizando de manera efectiva los conceptos y encontrándole significado a los mismos. Ausubel, (2002).

En cuanto a las habilidades procedimentales, se validó la hipótesis alterna para el grupo 1 puesto que en los resultados de la prueba postest se obtuvieron medianas más altas, esto por cuanto el simulador permitió que el estudiante realizara varios ensayos de tal manera que establecieran la mejor formulación, el orden de los ingredientes, y, el rango de temperatura y tiempo ideales para obtener un producto de buena calidad. Esto se explica porque según Shuell (1990) en la segunda fase del aprendizaje, los individuos toman la retroalimentación que reciben del resultado y la emplean para perfeccionar la estructura cognitiva haciendo el conocimiento, generalizado, abstracto y descontextualizado, y listo para ser usado en una situación específica o actividad de transferencia.

Los mejores resultados obtenidos por el grupo 1, en el bloque de preguntas de razonamiento, se debieron a que en la estrategia se le planteó al estudiante un problema de mediana complejidad en el que debía controlar variables y para ello tenía que analizar, sintetizar y transferir su conocimiento a la solución del problema. Esto permite afirmar que cuando un individuo es orientado para solucionar problemas debe construir varios modelos mentales para llegar a la solución, y si esto se hace durante varias sesiones de trabajo, se logra desarrollar mejores habilidades de razonamiento deductivo que cuando no se hace.

Para el Grupo 2, en los diferentes bloques de preguntas se asume la hipótesis nula, puesto que la prueba estadística demuestra que no hubo incidencia en el desarrollo conceptual, procedimental y de razonamiento de los estudiantes, a pesar de que los estudiantes manipularon el simulador. Esto reafirma lo planteado por diversos autores: los objetos virtuales como los simuladores son medios pedagógicos y no el fin en el sistema de aprendizaje, por lo tanto no promueven por sí solos las estructuras cognitivas.

Con estos resultados se comprobó que los estudiantes, a quienes se les orientó el aprendizaje a través de la estrategia, utilizaron todos los recursos del simulador motivando la reflexión y el análisis de los procedimientos que se necesitan para obtener un buen producto y resolver el problema planteado.

Con el fin de establecer si existieron diferencias significativas en el nivel de competencia alcanzado en la prueba de postest del grupo 1, se compararon las medianas a través del test de Kruskall Wallis. El valor obtenido de p fue $=0,0000404132$, lo que significa que hubo diferencias altamente significativas entre estas medianas, con un nivel de confiabilidad del 95\%. En el Gráfico 1 se puede observar que a pesar de que en los tres bloques de preguntas existieron diferencias estadísticas, donde se hicieron más evidentes estas diferencias fue en la adquisición de habilidades de tipo procedimental, y de desarrollo conceptual. Esto dice que el 
simulador orientado con la estrategia ABP tuvo mayor incidencia en estos aspectos y se pudo demostrar también que hay una correlación entre aprendizaje, adquisición y aplicación del conocimiento durante la solución del problema.

Gráfico 2. Diferencias significativas en las medianas de las pruebas realizadas por el Grupo 1

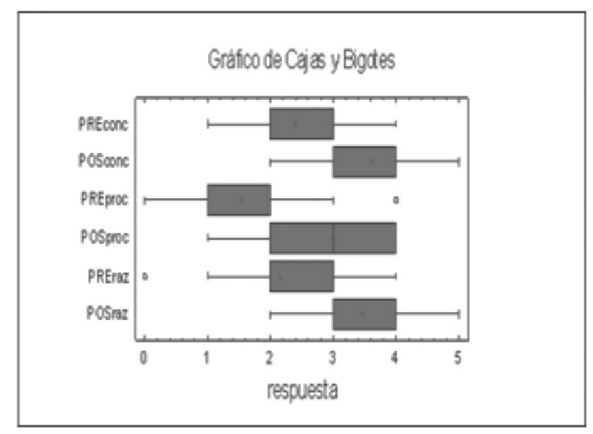

Fuente: Esta investigación.

\section{CONCLUSIONES}

En la investigación se pudo establecer que el desarrollo de actividades académicas en un escenario mediado con las TIC, orientado bajo una metodología desarrolladora como la ABP, despierta el interés del estudiante y favorece el desarrollo de competencias procedimentales como lo revelan los resultados del grupo 1, correspondiente a quienes realizaron la estrategia propuesta $\mathrm{ABP}$ y, más adelante, presentaron el postest con medianas más altas.

Esto nos permite reflexionar en el sentido de que en la educación a distancia mediada por tecnologías, se debe abandonar la idea de instrucción y fortalecer, por tanto, las investigaciones en didáctica que dinamicen el proceso de aprendizaje autónomo en los estudiantes que optan por esta modalidad.

El maestro-tutor debe ser un conocedor de los complejos procesos cognitivos que se realizan durante el aprendizaje, con el fin de que pueda orientar dicho proceso hacia el desarrollo de esas habilidades mentales de orden superior y pueda utilizar las TIC como herramientas efectivas y auxiliadoras de este proceso.

El desarrollo de las habilidades mentales de orden superior no se da en un proceso de aprendizaje espontáneo, sino, que este debe ser estimulado a través de entrenamiento formal, mediante actividades planificadas y orientadas hacia objetivos específicos de aprendizaje.

\section{REFEFENCIAS BIBLIOGRÁFICAS}

AUSUBEL, D. P. Adquisición y retención del conocimiento. Una perspectiva cognitiva. Barcelona: Ed. Paidós. 2002. 
BORRAS, I. Enseñanza y aprendizaje con la internet: una aproximación crítica, basada en simulaciones informáticas. Revista Electrónica de Enseñanza de las Ciencias, 5, (2).

COLL, C. Significado y sentido en el aprendizaje escolar. Reflexiones en torno al concepto de aprendizaje significativo. Barcelona: Paidós. 1990.

EISNER, E. Cognición y currículo: una visión nueva. Buenos Aires: Amorrortu, 1994.

ESTEBAN, M. El diseño de entornos de aprendizaje constructivista. En: Revista de Educación a distancia, Publicación en línea. Murcia (España). nro. 6. Noviembre de 2002.

ESTEBAN, M. La Educación a Distancia en la Sociedad del Conocimiento. UNESCO.

FAINHOLC, B. El concepto de mediación en la tecnología educativa apropiada y crítica. Portal educativo Educar. 2004.

GALVIS, A. Internet y aprendizaje. Experiencias y lecciones aprendidas. En: < http://www. metacursos.com> Consulta realizada en septiembre de 2008.

GARCÍA, A. y M. R. Gil. Entornos constructivistas de aprendizaje basado en simulaciones informáticas. En: Revista Electrónica de Enseñanza de las Ciencias Vol. 5, nro. 2. 2006.

GARGALLO, L. Estrategias de Aprendizaje. Centro de Investigaciones y documentación educativa. Universidad de Valencia. 2000.

GÓMEZ, G. Un modelo para la Enseñanza de las Ciencias. Revista Electrónica de Enseñanza de las Ciencias. Vol. 4 nro. 3. 2005.

HILERA, J; OTÓN, S. Y MARTÍNEZ, J. Aplicación de la realidad virtual en la enseñanza a través de internet. Cuadernos de documentación multimedia, 8. 1999. En: <http://www. ucm.es/info/multidoc/multidoc/revista/num8/index8.html>

LÓPEZ, M. Las TIC en la enseñanza de las ciencias. Los laboratorios virtuales. En: Revista Electrónica de Enseñanza de las Ciencias Vol. 6, nro. 3, 2002.

MARQUÉS G. Impacto de las TIC en la educación: funciones y limitaciones. (2000). DIM (Didáctica y Multimedia). En: http://dewey.uab.es/pmarques/dim/

MORTIMER, L. Objects of Desire: Promise and Practicality. Learning Circuits. Reference Center. Development of Educational Materials. Septiembre de 2008.

PONTES, A. Aplicaciones de las tecnologías de la información y de la comunicación en la educación científica. Segunda parte: aspectos metodológicos. En: Revista Eureka sobre Enseñanza y Divulgación de las Ciencias, 2(3). 2005. 
ROJAS, et al. Tendencias Educativas Contemporáneas. Universidad de la Habana. 2005.

SHUELL, T. J. Phases of meaningful learning. Review of educational research. 60. 1990.

SILVESTRE M. Aprendizaje, Educación y Desarrollo. Cuba: Editorial Pueblo y Educación. 1999.

VARELA, M. P. y MARTÍNEZ, M. M. Una estrategia de cambio conceptual en la enseñanza de la física: La resolución de problemas como actividad de investigación. En: Enseñanza de las Ciencias, 15(2). 1997.

ZAPATA, D. Contextualización de la educación virtual en Colombia. Medellín: Universidad de Antioquia. 2002. 